\title{
Finanzierung und Wohlstandsteilung als Tabuthemen
}

Marie Thorndahl

\section{(2) OpenEdition Journals}

Electronic version

URL: http://journals.openedition.org/sjep/567

DOI: $10.4000 /$ sjep.567

ISSN: 1663-9677

\section{Publisher}

Institut de hautes études internationales et du développement

\section{Printed version}

Date of publication: 1 novembre 2003

Number of pages: $207-213$

ISSN: $1660-5926$

\section{Electronic reference}

Marie Thorndahl, «Finanzierung und Wohlstandsteilung als Tabuthemen », Schweizerisches Jahrbuch für Entwicklungspolitik [Online], 22-2 | 2003, Online erschienen am: 10 Juni 2010, abgerufen am 08 September 2020. URL : http://journals.openedition.org/sjep/567 ; DOI : https://doi.org/10.4000/sjep. 567 


\section{Finanzierung und Wohlstandsteilung als Tabuthemen}

\section{Marie Thorndahl*}

D

ie von den Vereinten Nationen organisierten Gipfeltreffen stehen im Ruf, wegen der Diskrepanz zwischen den Absichtserklärungen und der Verwirklichung von Grundsätzen und Aktionsplänen Enttäuschung hervorzurufen. Wenn die Staaten die Unterlagen einmal verabschiedet haben, verknüpfen sie sie nur selten mit angemessenen Finanzierungen, was zwischen den Ländern des Nordens und des Südens sowie bei der Zivilgesellschaft starke Spannungen auslöst. Eine gerechte Informationsgesellschaft erfordert neue Ressourcen; die Verwirklichung des Aktionsplans des Weltgipfels über die Informationsgesellschaft (WSIS) steht einmal mehr vor einem finanziell stark angespannten Hintergrund.

Zunächst verfügen die Staaten im Norden wie im Süden über geringere Kapazitäten für die Mobilisierung der Ressourcen. Nur wenige neue öffentliche Finanzierungsmechanismen wurden angesichts der Globalisierung und der Deregulierung der nationalen Volkswirtschaften geschaffen. Die Beitragssätze sinken, und auf zahlreiche Wirtschaftsaktivitäten wird keine Steuer erhoben (Reichtum im Zusammenhang mit den elektronischen Netzen, Paradoxon von Solow ${ }^{1}$, Steuerparadiese, Steuerkonkurrenz usw.).

Auch die der öffentlichen Solidarität zugewiesenen Mittel sind im Rückgang begriffen. Trotz der Absichtserklärungen - gemäss den Empfehlungen der Vereinten Nationen sollen $0,7 \%$ des BSP für die öffentliche Entwicklungshilfe (ODA) verwendet werden - verzeichnen die Beiträge der Staaten einen beispiellosen Tiefstand: Zwischen 1990 und 1999 sank die ODA von 0,34 auf $0,24 \%$ des BSP der Geberländer ${ }^{2}$.

* Sozioökonomin, Frankreich.

1 Nach Robert Solow, der bereits in den 50er-Jahren die Bilanz zog, dass Computer überall - ausser in den Statistiken über die Produktivität - anzutreffen seien. Robert Solow, „Technical Change and the Aggregate Production Function“, Review of Economics and Statistics, Nr. 39, 1957, S. 312-320. Durch elektronische Netze erzeugte Einsparungen und Reichtum (Produktivitätsgewinne, sinkende Transport- und Lagerkosten, Verkauf neuer Produkte usw.) werden kaum auf die Verbraucherpreise und auf Lohnerhöhungen überwälzt. Sie konzentrieren sich vor allem in privaten Wirtschaftseinheiten ohne Auswirkungen für die Gemeinschaft. Im übrigen bedeutet der E-Commerce eine Gefahr für die Verbrauchssteuer (wie z.B. die Mehrwertsteuer), denn der Cyberspace untersteht keiner Regierung und entgeht häufig jeglicher Besteuerung. Zwar setzen sich die Staaten (vor allem die Europäische Gemeinschaft) allmählich mit dem Problem auseinander, aber die Fragen sind nach wie vor sehr umstritten. Der Cyberspace bleibt grösstenteils ein steuerfreier Raum.

2 UNDP, Rapport mondial sur le développement humain 2001. Mettre les nouvelles technologies au service du développement, New York; Oxford, 2001, S. 190. 
Als der WSIS in den Jahren 1998-1999 konzipiert wurde, glaubten die Initianten, die Quadratur des Kreises gefunden zu haben. Die ungebremste Expansion des Nasdaqs weckte die Hoffnung, dass die Sponsoren scharenweise anklopfen würden - nach dem Modell der Telecom ${ }^{3}$, dem von der Internationalen Fernmeldeunion (ITU) ausgerichteten High-Tech-Messe - und dass der Geldsegen auch die Finanzierung neuer Projekte und Aktionspläne erleichtern würde. Die Börsenkräche und Konkurse der Jahre 2000-2002 haben jedoch dieses Szenario ruiniert. Der WSIS ringt sogar nach Mitteln, um die eigentliche Veranstaltung zu finanzieren.

Welche Vorschläge könnten angesichts dieser Verhältnisse die Verwirklichung eines Aktionsplans garantieren? Welche politischen Öffnungen zeichnen sich $\mathrm{ab}$, um innerhalb von WSIS originelle Lösungen zu entwickeln?

\section{Viele Ideen, wenig Forschung}

Die - zwar sehr unterschiedlichen - Vorschläge für Alternativfinanzierungen lassen sich unter dem allgemeinen Terminus ,globale Abgaben“ zusammenfassen: Es geht darum, eine international verwaltete Steuer zu erheben, um damit auf globale Probleme zu reagieren. Dabei werden die gleichen Ziele verfolgt wie mit der nationalen Besteuerung: Durch Anreize sollen bestimmte schädliche Praktiken vermieden (Verschmutzung, Spekulationen usw.), Verhaltensweisen geändert und Ressourcen umverteilt werden, um globale öffentliche Güter oder internationale Solidaritätsprogramme zu finanzieren.

Die Vereinten Nationen und verschiedene Bürgerbewegungen haben in diesen Bereichen seit den 70er Jahren eine Fülle von Vorschlägen unterbreitet: Besteuerung des Flugverkehrs, der internationalen Fischerei, der Waffen, der Kohlendioxidemissionen usw. Nur wenige Vorschläge wurden indessen vertieft prospektiv untersucht und diskutiert. Wie Luc Soete für die bit tax feststellt, liegt hier ein no man's land der Forschung, weil die Fragen tendenziöse Kontroversen hervorrufen, welche die Forschung belasten. Das Interesse für globale Abgaben an sich würde bereits falsche Signale an die Investoren aussenden und die Volkswirtschaften gefährden 4 . Seit den 90er Jahren - und nach dem Druck des amerikanischen Kongresses - liessen die Vereinten Nationen verschiedene Forschungsprojekte über globale Abgaben fallen 5 .

$<$ www.itu.int/WORLD2003>.

Luc Soete und Karin Kamp, The Bit Tax: The Case for Further Research, Universität Maastricht, August 1996, S. 2; International Communication Round Table, Position Paper on the „Bit Tax“, Brüssel, Juli 1997.

5 Jens Martens und James A. Paul, The Coffers Are not Empty: Financing for Sustainable Development and the Role of the United Nations, Juli 1998, <www.globalpolicy.org/sececon/global/ paul.htm>. Die Studien werden jedoch je nach Vorschlag unterschiedlich vertieft: Die meisten Überlegungen und Diskussionen wurden zu den Ökoabgaben (Grundsatz des Verursacherprinzips) und zur Abgabe zur Eindämmung der Finanzspekulationen geführt. 
In der Folge soll ein kurzer Überblick über die vorgeschlagenen Abgaben vermittelt werden, die direkt auf den neuen Informations- und Kommunikationstechnologien beruhen.

Die einzige mit den elektronischen Netzen verbundene Abgabe ${ }^{6}$, die weltweit diskutiert und studiert wurde, ist die Abgabe auf Finanztransaktionen (besser bekannt unter der Bezeichnung Tobin-Steuer). Wenn ein minimaler Prozentsatz auf Devisentransaktionen erhoben würde, liessen sich Spekulation und Geldvolatilität verringern und umfassende Ressourcen für die internationale Solidarität freisetzen. Es handelt sich um eine Korrektur- und Umverteilungssteuer und gleichzeitig um eine symbolische Steuer, die beweisen würde, dass das internationale Finanzsystem reguliert werden kann, vorausgesetzt, der politische Wille ist vorhanden. Die Tobin-Steuer hat in fachlicher und politischer Hinsicht an Glaubwürdigkeit gewonnen. Die Steuer wird von zahlreichen Regierungs- und Parlamentsvertretern sowie von Gewerkschaften und Intellektuellen unterstützt. Der Internationale Währungsfonds (IWF) und andere Einrichtungen, welche die Steuer kategorisch ablehnten, räumen mittlerweile ein, dass sie unbestrittene Vorteile besitzt, insbesondere nach den Lobesworten in der Studie, die Paul Bernd Spahn - ehemaliger Volkswirtschaftler beim IWF - im Auftrag des deutschen Bundesministeriums für wirtschaftliche Zusammenarbeit und Entwicklung durchführte ${ }^{7}$.

Ein weiterer Vorschlag, der direkt auf die Expansion des Internets zurückgeht, ist die bit tax. Sie bestünde darin, eine geringe, am Internet-Verkehrsvolumen indexierte Abgabe zu erheben. Der Vorschlag wurde vom Club of Rome im Jahr 1994 entwickelt und vom Entwicklungsprogramm der Vereinten Nationen (UNDP) verbreitet (der Vorschlag wird im UNDP-Jahresbericht 1999 erläutert $)^{8}$. Es handelt sich um die einzige globale Abgabe, die spezifisch zur Bekämpfung der digitalen Kluft entwickelt wurde. Der Vorschlag löste jedoch vehementen Widerstand aus und wurde praktisch im Keim erstickt. Gemäss einem UNDP-Bericht drohte der amerikanische Kongress mit einem Rückzug der Vereinigten Staaten aus den Vereinten Nationen, wenn dort weiterhin über Vorhaben für globale Steuern diskutiert werde ${ }^{9}$.

Der zweite Vorschlag des UNDP, der im Jahresbericht 2001 erörtert wird, betrifft die exit tax auf den Braindrain. Diese Steuer ist eine Reaktion auf die Politik der Arbeitskräfteimporte der Länder des Nordens zur Förderung ihrer Informatikindustrie ${ }^{10}$. Schätzungen des UNDP zufolge verliert Indien allein bei

6 Der exponentielle Anstieg der Finanztransaktionen und der Spekulation wurde durch die elektronischen Netze erst ermöglicht. Die technische Machbarkeit der Abgabe beruht auf der Einführung von elektronischen Verfahren in Banken, die Devisengeschäfte durchführen dürfen.

7 Paul Bernd Spahn, On the Feasibility of a Tax on Foreign Exchange Transactions, Report commissioned by the Federal Ministry for Economic Cooperation and Development, Bonn, Januar 2002, $<$ http://much-magic.wiwi.uni-frankfurt.de/professoren/spahn/tobintax >.

8 UNDP, Human Development Report 1999, New York; Oxford, 1999, S. 66.

9 Prohibition on United Nations Taxation Act of 1999 (Introduced in the House), 106. Kongress, 6. Januar 1999, <www.globalpolicy.org/socecon/glotax/biblio/uscong.htm>.

10 Im Jahr 2000 verabschiedeten die Vereinigten Staaten neue Gesetze für den „Import“ von 200’000 Fachkräften pro Jahr (im wesentlichen im Bereich Informatik und neue Technologien). Einige euro- 
den Ausbildungskosten für die abgewanderten Fachleute jährlich 2 Milliarden US-Dollar. Die exit tax in Höhe von zwei Monatsgehältern würde dem Land 1 Milliarde Dollar jährlich einbringen, wodurch der nationale Etat für die höhere Bildung um rund ein Drittel erhöht werden könnte ${ }^{11}$. Allerdings wurde der Vorschlag nicht zur Speisung eines internationalen Solidaritätsfonds konzipiert. Deshalb wäre er für die Einführung eines globalen Aktionsplans wenig sinnvoll.

Weitere Ideen bestehen darin, die Domain-Namen zu besteuern: entweder auf nationaler Ebene (zum Beispiel .ch) oder auf internationaler Ebene (zum Beispiel .org oder .com). Diese Vorschläge widersprechen sich häufig und stossen bei den Internet-Pionieren und den Internet-Nutzern auf Widerstand. Die Hauptargumente für eine Steuer auf dem Verkauf von Domain-Namen beruhen auf dem Prinzip der globalen öffentlichen Güter. Als gemeinsame Ressource müssen die Domain-Namen vor Spekulanten geschützt werden; gleichzeitig sollen Ressourcen frei werden, um die nicht-gewinnorientierten Sektoren der südlichen Länder bei der Entwicklung ihrer Netze zu unterstützen ${ }^{12}$. Eine Premiere bildet die Internet Society: Seit 2003 verwaltet die Nichtregierungsorganisation die Extension .org und verfolgt den Zweck, den Mehrwert für die Ausbildung und Entwicklung des Internets in den Ländern des Südens einzusetzen.

Ein weiterer Vorschlag wäre, die Nutzung radioelektronischer Frequenzspektren zu besteuern. Die internationale Fernmeldeunion (ITU) ist für die Bewirtschaftung der radioelektronischen Frequenzspektren und für die Satellitenumlaufbahnen zuständig. Dabei handelt es sich um natürlich begrenzte Ressourcen, für welche zahlreiche Dienstleistungen eine steigende Nachfrage anmelden: Festnetz- und Mobiltelefonie, Rundfunk, Weltraumforschung, Meteorologie, globale Funkortungssysteme, Umweltüberwachung, Kommunikationsdienste für die Überwachung des Meeres- und Luftraumes usw. Die Aufteilung der Frequenzen ist derzeit eine kostenlose Dienstleistung der ITU; sie könnte die Frequenzen den Regierungen verkaufen, die sie anschliessend selbst aufteilen würden. In zahlreichen Ländern wird das Management der Spektren bereits national besteu$\operatorname{ert}^{13}$. Es fragt sich, ob diese Praxis verallgemeinert werden soll, und ob der Erlös teilweise internationalen Projekten zufliessen soll ${ }^{14}$.

\section{Aus den Augen mit den Steuern!}

Trotz dreissig Jahren Diskussionen und Kontroversen über globale Abgaben werden nur minimale Fortschritte verzeichnet. Weder ein Staat noch eine Staatengruppe hat die Initiative ergriffen, um die Diskussion in Instanzen zu tragen,

päische Länder und Japan verfolgen die gleiche Politik. Allein in den Vereinigten Staaten wird die Hälfte aller Visa an indische Informatiker ausgestellt.

11 UNDP, Rapport mondial sur le développement humain 2001, op. cit., S. 92.

12 Die Regel des „first come, first served“ benachteiligt diejenigen, die spät zum Internet kommen insbesondere die Länder des Südens. Die Entwicklung von IPV6, der neuen Internet-Generation, dürfte indessen das Problem der Knappheit der Domain-Namen sowie dasjenige der Kontrolle der Vereinigten Staaten über die Mehrheit der Namen beseitigen.

13 Internationale Fernmeldeunion, Financement de la gestion du spectre: principes, Januar 1999, verfügbar auf der Website der ITU <www.itu.int $>$.

$14<$ <ttp://user.intersatx.net/jc/campaign_finance.html>. 
die gegebenenfalls für die Verwirklichung der Projekte zuständig wären. Auch die jüngste Vergangenheit rät zur Vorsicht.

Ein historischer Abriss dazu: Der Millenniums-Gipfel (New York, 2000), der für WSIS eine ständige Inspirationsquelle bedeutet, hatte die Finanzierungsfragen über Bord geworfen und gleichzeitig hochfliegende Ambitionen betreffend die Zielvorgaben verkündet. Die Konferenz der Vereinten Nationen über Finanzierung und Entwicklung, die ein Jahr später in Monterrey tagte, war eigens dafür einberufen worden, diese Probleme anzugehen. Die Konferenz scheiterte weitgehend an dieser Aufgabe, und die Frage der globalen Abgaben wurde quasi tabuisiert. Nach Auffassung des Vorbereitungsausschusses, der sich mit der Schaffung einer internationalen Organisation für Steuerfragen befasst ${ }^{15}$, war die Zeit noch nicht reif, um eine internationale Einigung über die Besteuerung der multinationalen Konzerne und über die globalen Abgaben anzustreben. Obwohl die alternativen Finanzierungen also nicht auf der Tagesordnung standen, werden sie in verschiedenen vorbereitenden Dokumenten, darunter im Zedillo-Bericht, erwähnt ${ }^{16}$.

Letztlich fiel der Konsens von Monterrey sehr konservativ aus: Die öffentliche Entwicklungshilfe bleibt das staatliche Finanzierungsinstrument par excellence. Der Konsens appelliert an alle Länder, Anstrengungen mit Blick auf die Zielvorgabe von $0,7 \%$ zu leisten, ohne dass die Staaten indessen feste Verpflichtungen übernehmen. Ausserdem wird im Konsens bekräftigt, dass Freihandel und Investitionsflüsse für die ärmsten Länder weiterhin den Schlüssel zum Erfolg bedeuten, vorausgesetzt, dass diese sich die Möglichkeit verschaffen, davon zu profitieren.

Die Einführung von Abgaben auf die Nutzung elektronischer Netze scheint deshalb unwahrscheinlich, da die Staaten sich mit der Unterzeichnung von Steuerbefreiungs-Abkommen innerhalb der Welthandelsorganisation (WTO) gleichsam selbst die Hände gebunden haben. Seit 1998 haben 132 Staaten in der WTO eine Ministererklärung angenommen: das Standstill Agreement for Duty-free Cyberspace. Dieses ,gentleman's agreement“ über die Steuerbefreiung elektronischer Transaktionen wurde im Jahr 2001 in Doha verlängert. Ausserdem nahmen im Februar 200257 WTO-Mitglieder, die zusammen $93 \%$ des Handels mit Produkten der Informations- und Kommunikationstechnologie ausmachen, das Information Technology Agreement (ITA) an. Sie verpflichten sich mit diesem Abkommen, die Abgaben auf eine lange Liste von Produkten abzubauen ${ }^{17}$. Schliesslich werden die meisten Transaktionen, die den elektronischen Handel betreffen, in das Allgemeine Abkommen über den Dienstleistungshandel (GATS), das derzeit in der WTO ausgehandelt wird,

15 Bestimmte Kreise vertreten die Meinung, die internationale Organisation für Steuerfragen könnte auch globale Abgaben erheben (Tobin-Steuer oder carbon tax) und sich für die Verringerung des Steuerwettbewerbs unter den Ländern einsetzen; <www.newsmax.com/archives/articles/2002/1/2/ 63214.shtml>.

16 <www.un.org/esa/ffd/a55-1000.pdf>. Anlässlich dieser Konferenz wurde nachdrücklich bekräftigt, dass der internationale Handel der Motor für Wachstum und Entwicklung sei. Vereinte Nationen, Consensus de Monterrey, März 2002, <www.un.org/esa/ffd/aconf198-3-f.pdf>.

17 UNCTAD, E-commerce and Development Report 2002, New York; Genf, 2002, S. 110-111. 
übernommen; Ziel ist einmal mehr die Verringerung der Steuern. Diese Bestimmungen schränken den Spielraum für künftige Optionen und Beschlüsse, die im Rahmen von WSIS getroffen werden können, bereits erheblich ein.

\section{Von der Steuer zu Netaid}

Erst anlässlich des zweiten Vorbereitungstreffens (PrepCom 2) im Februar 2003 wurde das heikle Thema der Finanzierung des Aktionsplans von WSIS angesprochen. Der senegalesische Präsident Abdoulaye Wade plädierte als erster für die digitale Solidarität, mit welcher die internationale Staatengemeinschaft sich verpflichten würde, alle Länder innerhalb einer „,digitalen Schlange“ (d.h. innerhalb einer oberen und unteren Konnektivitäts-Bandbreite) zusammenzuführen, um die Ungleichheiten langfristig zu beseitigen ${ }^{18}$. Nach Auffassung der senegalesischen Initianten könnten diese Bemühungen gemeinsam von den Verbrauchern, Unternehmen und Staaten finanziert werden, wobei insbesondere eine Abgabe auf den Verkauf von Informatikmaterial (beispielsweise 1 Dollar pro gekauften oder verkauften Computer) erhoben würde.

Der WSIS-Kontext könnte diesem Vorschlag unter bestimmten Voraussetzungen förderlich sein. Die Unternehmen suchen händeringend nach neuen Absatzmärkten. Die paar Dollar Abgaben auf ihren Umsatz könnten eine Multiplikatorwirkung erzeugen und sich - nachdem sie das internationale System passieren, in welchem eine Abschöpfung stattfindet - künftig in Aufträge verwandeln. Insofern entspricht die Abgabe auf Ausstattungen dem Win-WinMythos, wonach die Unternehmen des Nordens sich neue Absatzmärkte erschliessen, indem sie den insolventen Bewohnern des Südens einen InternetAnschluss bereitstellen. Dieser - völlig entpolitisierte - Vorschlag besitzt nur insoweit Erfolgschancen, als er sich von der globalen, auf obligatorischen Abgaben beruhenden Steuer abhebt. Falls die numerische Solidarität durch freiwillige Beiträge genährt werden soll, so wird dieser Vorschlag anderen Initiativen der Vereinten Nationen wie NetAid ${ }^{19}$ oder Global Compact ${ }^{20}$, die auf Schenkungen von Unternehmen, Staaten und Einzelpersonen beruhen, und die sich im Kampf gegen die globalen Ungleichgewichte keineswegs bewährt haben, zum Verwechseln ähnlich sehen.

Die freiwilligen Finanzierungsmechanismen bilden den Gegenpol zu den Forderungen der Bürgerbewegungen, die sich für eine tiefgreifende Neuverteilung des Wohlstandes einsetzen. Danach könnten nur eine Devisentransaktionssteuer und der Schuldenerlass für Länder des Südens (Hand in Hand mit dem Kampf gegen die Steuerparadiese und die Steuerkonkurrenz) signifikante Fortschritte erlauben. Allerdings ist es unwahrscheinlich, dass diese Forderungen die Diskussionen im Rahmen des WSIS beeinflussen. Die akkreditierte Zivilgesellschaft ist mit internen Spannungen und dem Ringen um kurzfristige Finanzmittel, welche die eigene Teilnahme an den Arbeiten des Gipfels garantieren sollen, ausgelas-

\footnotetext{
18 Abdouaye Wade, Wortmeldung vom 17. Februar 2003, PrepCom 2, Genf, <www.itu.int/wsis/docs/ pc2/visionaries/wade-fr.doc $>$.

$<$ www.netaid.org $>$.

$20<$ www.unglobalcompact.org $>$
} 
tet. Sie schont ihre Partner und identifiziert sich nur bedingt mit den als radikal geltenden Bewegungen, die sich in Porto Alegre oder Evian zu Wort melden.

Die von den WSIS-Teilnehmern propagierten Lösungen - insbesondere öffentlich-private Partnerschaften, öffentlich-öffentliche Partnerschaften, Direktinvestitionen oder die dezentralisierte Entwicklungszusammenarbeit - bieten sicherlich einige interessante Perspektiven für die Projektfinanzierung an. Alle Vorschläge weichen jedoch der Grundsatzfrage der Aufteilung des Reichtums in der Informationsgesellschaft aus. Der Appell, bis zum Jahr 2010 alle Dörfer der Welt zu vernetzen ${ }^{21}$, wird - wie das 1979 verkündete Ziel „Gesundheit für alle bis zum Jahr 2000“22 - eine weitere moderne Prophezeiung (mit denen uns die Gipfel überschütten, aber an die niemand mehr wirklich glaubt) bleiben.

21 WSIS (World Summit on the Information Society), Draft Action Plan: Based on Discussions in the Working Group of Sub-Committee 2, Dokument WSIS/PCIP/DT/2-E, 21. März 2003, S. 8, verfügbar auf der Website der ITU <www.itu.int>.

22 Die Stratégie mondiale de la santé pour tous d'ici l'an 2000 wurde anlässlich der 32 . Weltgesundheitsversammlung im Jahr 1979 (ausgerichtet von der Weltgesundheitsorganisation) lanciert; $<$ www.who.int $>$. 